\title{
Genome-wide association of major depression: description of samples for the GAIN Major Depressive Disorder Study: NTR and NESDA biobank projects
}

\author{
Dorret I Boomsma*, ${ }^{*}$, Gonneke Willemsen ${ }^{1}$, Patrick F Sullivan ${ }^{2}$, Peter Heutink ${ }^{3}$, Piet Meijer ${ }^{4}$, \\ David Sondervan ${ }^{3}$, Cornelis Kluft ${ }^{4}$, Guus Smit ${ }^{5}$, Willem A Nolen ${ }^{6}$, Frans G Zitman ${ }^{7}$, \\ Johannes H Smit ${ }^{8}$, Witte J Hoogendijk ${ }^{8}$, Richard van Dyck ${ }^{8}$, Eco JC de Geus ${ }^{1}$ and \\ Brenda WJH Penninx $6,7,8$
}

\footnotetext{
${ }^{1}$ Netherlands Twin Register, Department of Biological Psychology, VU University Amsterdam, Amsterdam, Netherlands; ${ }^{2}$ Department of Genetics, University of North Carolina at Chapel Hill, Chapel Hill, NC, USA; ${ }^{3}$ Medical Genomics Section, Department of Clinical Genetics, VU Medical Center, Amsterdam, Netherlands; ${ }^{4}$ Gaubius Laboratory, TNO-PG, Leiden, Netherlands; ${ }^{5}$ Department of Molecular and Cellular Neurobiology, Faculty of Earth and Life Sciences, VU University Amsterdam, Amsterdam, Netherlands; ${ }^{6}$ Department of Psychiatry, University Medical Center Groningen, University of Groningen, Groningen, Netherlands; ${ }^{7}$ Department of Psychiatry, Leiden University Medical Center, Leiden, Netherlands; ${ }^{8}$ Department of Psychiatry, EMGO Institute, Institute of Neuroscience, VU Medical Center, Amsterdam, Netherlands
}

To identify the genomic regions that confer risk and protection for major depressive disorder (MDD) in humans, large-scale studies are needed. Such studies should collect multiple phenotypes, DNA, and ideally, biological material that allows gene expression analysis, transcriptomic, proteomic, and metabolomic studies. In this paper, we briefly review linkage studies of MDD and then describe the large-scale nationwide biological sample collection in Dutch twin families from the Netherlands Twin Register (NTR) and in participants in the Netherlands Study of Depression and Anxiety (NESDA). Within these studies, 1862 participants with a diagnosis of MDD and 1857 controls at low liability for MDD have been selected for genome-wide genotyping by the US Foundation for the National Institutes of Health Genetic Association Information Network. Stage 1 genome-wide association results are scheduled to be accessible before the end of 2007. Genome-wide association results are open-access and can be viewed at the dbGAP web portal (http://www.ncbi.nlm.nih.gov). Approved users can download the genotype and phenotype data, which have been made available as of 9 October 2007.

European Journal of Human Genetics (2008) 16, 335-342; doi:10.1038/sj.ejhg.5201979; published online 16 January 2008

Keywords: major depression; GAIN; biological sample collection; genome-wide association analysis; linkage review

\footnotetext{
*Correspondence: Professor DI Boomsma, Department of Biological Psychology, VU University, Van der Boechorststraat 1, Amsterdam 1081 BT, The Netherlands.

Tel: + 3120598 8787; Fax: + 3120598 8832;

E-mail: dorret@psy.vu.nl

Received 26 June 2007; revised 13 November 2007; accepted 15 November 2007; published online 16 January 2008
}

Introduction

The defining features of major depressive disorder (MDD) are marked and persistent depressed mood, associated with physical and cognitive signs and symptoms (anhedonia, insomnia or hypersomnia, appetite and weight changes, psychomotor agitation or retardation, fatigue or loss of energy, excessive guilt or worthlessness, poor 
concentration or indecisiveness, and recurrent thoughts of death or suicide). MDD is distinct from 'normal' sadness by its persistence (ie, $\geq 2$ weeks), the presence of accompanying signs and symptoms, and its associated impairment. The definition of MDD excludes other disorders that can have substantial depressive symptoms. These include bipolar disorder, schizophrenia, schizoaffective disorder, and depressive symptoms resulting from another disorder (eg, medical condition like hypothyroidism). ${ }^{1}$

Combining available estimates, the lifetime prevalence of $\mathrm{MDD}$ is $\sim 15 \%$ and is twofold higher in women than in men. ${ }^{2}$ The course of MDD is typified by recurrence of illness. In a meta-analysis, $76 \%$ had $\geq 1$ recurrence over a 10-year follow-up. ${ }^{3}$ MDD is associated with large morbidity (greater than chronic medical conditions such as diabetes and arthritis), ${ }^{4-6}$ excess mortality from suicide and other causes, ${ }^{7-10}$ and substantial direct and indirect costs $(>\$ 43$ billion/year in the US). ${ }^{11}$ The WHO projected MDD to be the second leading cause of disability worldwide by $2020 .{ }^{12}$ Thus, MDD is a first-rank public health problem.

It has been firmly established that variation in MDD liability is in part genetic. ${ }^{13}$ Family studies find a significantly higher lifetime prevalence of MDD in biological relatives of MDD probands (pooled odds ratio $=2.84$, $95 \% \mathrm{CI}=2.31-3.49)$. Twin studies have established that the familial component reflects genetic vulnerability rather than shared environmental risk. The meta-analytic heritability estimate is $37 \%(95 \% \mathrm{CI}=31-42 \%)$ with a minimal contribution of environmental effects common to siblings $(0,95 \% \mathrm{CI}=0-5 \%)$, and substantial individual-specific environmental effects/measurement error (63, 95\% $\mathrm{CI}=58-67 \%)$.
Despite the evidence for heritability of MDD, as with most complex traits, identification of vulnerability genes has not been yet very successful. Results from studies of first-stage genome-wide linkage scans for MDD or related personality traits are summarized in Figure 1. One study was of $\mathrm{MDD},{ }^{14}$ two of recurrent $\mathrm{MDD},{ }^{15,16}$ and three of recurrent, early-onset MDD. ${ }^{17-19}$ Five studies investigated quantitative traits related to MDD, that is harm avoidance $^{20}$ and neuroticism. ${ }^{21-24}$ Two studies were secondary analyses of cohorts selected for alcoholism ${ }^{14}$ or nicotine dependence. $^{23}$

Results from linkage studies of MDD and associated personality traits are summarized in Figure 1. Regions on chromosomes $1,4,5,7,8,11,12$, and 13 show a significant linkage signal $(\mathrm{LOD}>3)$ in at least one study. However, taken together these studies do not indicate a large degree of replication. This lack of success so far, may be due to the relatively small sample sizes of most linkage studies. Likewise, the generally small sizes, the different definitions of the phenotype, and the limited number of polymorphisms that could be included in association studies of candidate genes have not lead to consistent results. This is even true for candidate genes that have been assessed in large sized studies. $^{25-27}$

However, since 2000 there have been exceptional advances in our knowledge of the human genome along with a precipitous drop in the cost of genotyping. These advances directly led to genome-wide association studies whereby large case-control samples are individually genotyped for 500000 or more single nucleotide polymorphisms. Examples where genome-wide association studies has provided new genetic insights include the

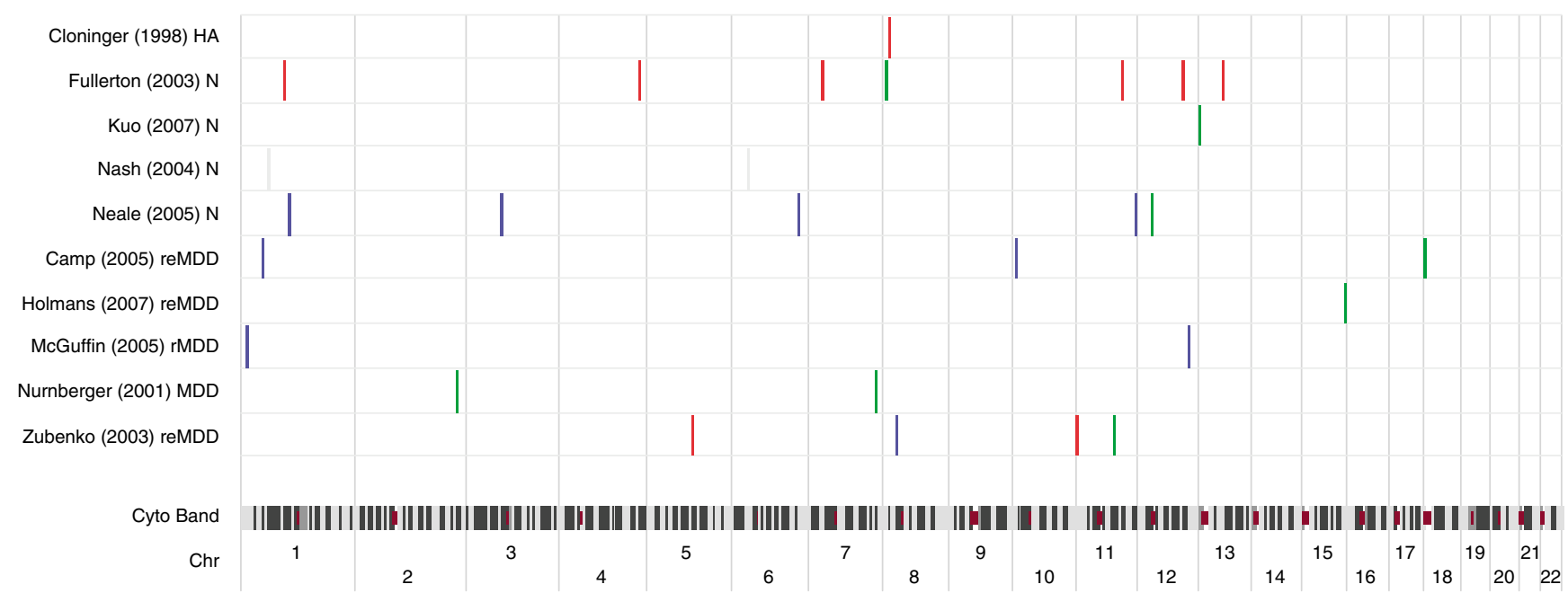

Figure 1 First-stage genome-wide linkage scans for MDD or related personality traits (neuroticism and harm-avoidance). The $x$-axis shows the human genome from 1 ptel to 22 tel. Each row shows the findings for that study. Studies of bipolar disorder, candidate region linkage studies, finemapping results, and statistical analyses of epistasis or studies that included covariate models were excluded. The width of the coloured bars indicates the genomic physical distance/region implicated by a particular study. LOD/equivalents are plotted. Key: red LOD $\geq 3$, green $L O D \geq 2$, blue $L O D \geq 1.5$, grey otherwise. 
CFH gene and age-related macular degeneration, ${ }^{28}$ the FTO gene and body mass index in children and adults, ${ }^{29}$ the TCF7L2 gene and type- 2 diabetes $^{30}$ and the IL23R gene and inflammatory bowel disease. ${ }^{31}$ Recently, the Wellcome Trust Case Control Consortium ${ }^{32}$ identified 24 association signals for seven major diseases, including bipolar disorder, using 2000 cases and 3000 controls.

In 2006, a consortium of investigators at the VU University Amsterdam, universities in Groningen and Leiden, and UNC-Chapel Hill in the US was selected for GWA genotyping as one of the six Genetic Association Information Network (http://www.fnih.org/GAIN) studies. ${ }^{33}$ The GAIN initiative is a component of the publicprivate partnership of the Foundation for the National Institutes of Health Inc., and funding is via Pfizer, Affymetrix, and Abbott Laboratories. GWA genotyping for 600000 single nucleotide polymorphisms in MDD cases and population-based controls has been conducted by Perlegen Sciences. Stage 1 genome-wide association results are scheduled to be available before the end of 2007 and will be available to the scientific community via the NCBI dbGAP web portal (http://www.ncbi.nlm.nih.gov). Approved users can download genotype and phenotype data with the restriction that use of genotype and phenotype data is restricted to psychiatric health and related somatic conditions.

In this paper, we describe the approach and logistics of the two large-scale projects in the Netherlands collecting biological samples for genetic studies, which together constitute the GAIN-MDD study. We give an overview of the phenotyping for MDD and the selection of case and control subjects for GWA genotyping in the GAIN-MDD study.

\section{Subjects}

Subjects eligible for inclusion in the GWA study of MDD come from two large longitudinal projects: 1702 depressed cases come from the Netherlands Study of Depression and Anxiety (NESDA, www.nesda.nl), and 1700 controls come from the Netherlands Twin Registry (NTR, www. tweelingenregister.org). In addition, 160 cases from the NTR and 157 controls from the NESDA study were included in the original selection, and both parents of 33 controls to form 33 trios and 21 duplicate samples.

\section{MDD cases}

Depressed subjects (cases) are mainly derived from NESDA, a longitudinal cohort study designed to be representative of those with depression and anxiety disorders in different health care settings and in different stages of the developmental history of the disorders. Details on objectives, recruitment, and methods of NESDA have been described elsewhere. ${ }^{34}$ In short, recruitment of participants took place from September 2004 through February 2007. Cases from mental health care organizations were recruited from seven outpatient regional facilities in the Netherlands (total catchment area $=1175000$ persons). When new patients were diagnosed with a depressive and/or anxiety disorder during a standardized intake assessment, they were asked to participate in NESDA. For recruitment of respondents in primary care, a three-stage screening procedure was used. A random sample of patients who consulted a GP in the last 4 months, irrespective of the reason for consultation, filled out a screening questionnaire. ${ }^{35}$ Those who screened positive were interviewed by phone with the short-form of the Composite International Diagnostic Interview (CIDI). ${ }^{36}$ Those with current MDD or an anxiety disorder were asked to participate in NESDA and were invited for a baseline assessment, which included a full CIDI interview. A community sample of NESDA cases was derived from the Netherlands Mental Health Survey and Incidence Study, a community-based study examining the prevalence of psychiatric disorders in the Netherlands. ${ }^{37}$ A multistage, stratified, random sampling procedure recruited 7076 respondents from different households in 90 Dutch municipalities. Those who were diagnosed with lifetime MDD or anxiety disorder during one of the CIDI interviews in 1996, 1997 or 1999, were approached for participation in NESDA. Finally, the NESDA sample includes a subgroup previously recruited for the Adolescents at Risk for Anxiety and Depression Study, ${ }^{38}$ a prospective cohort study examining the development and course of MDD and anxiety disorders among the offspring (18-25 years) of parents with depressive or anxiety disorders. Potential cases in the NTR were identified based on a multivariate composite score that combined survey data on depression, anxiety, and neuroticism. ${ }^{39}$ From this group of potential cases, we selected unrelated subjects, who received a CIDI interview by telephone.

Regardless of recruitment setting, similar inclusion, and exclusion criteria were used to select MDD cases. Inclusion criteria were a lifetime diagnosis of DSM-IV MDD as diagnosed through the CIDI psychiatric interview (section E, version 2.1), an age between 18 and 77 years and selfreported western European ancestry. Persons who were not fluent in Dutch and those with a primary diagnosis of a psychotic disorder, obsessive compulsive disorder, bipolar disorder, or severe substance use dependence were excluded. The 1862 cases included in GAIN, were recruited through mental health care organizations (785), primary care practitioners (603), and community samples (218 Netherlands Mental Health Survey and Incidence Study, 96 Adolescents at Risk for Anxiety and Depression Study and 160 NTR).

\section{Control subjects}

Control subjects are mainly derived from the NTR, which has collected longitudinal data by mailed surveys every 2-3 years since 1991. Data collection has occurred in 1991, 
1993, 1995, 1997, 2000, 2002/3, and 2004/5 and takes place in twins and their family members. There are nearly 22000 participants from 5546 families (some families are linked and come from larger pedigrees). The majority of families were recruited when the twins were young adults through City Council registration systems in 1990-91 and in 1992-93. After 1993 an effort was made to recruit adult and older twins through a variety of approaches. Details on recruitment, response rates, response bias, and demographic characteristics of the sample have been described previously. ${ }^{40}$ The sex distribution is $40 \%$ men and $60 \%$ women for twins, 45 and $55 \%$ for the siblings of twins, 47 and 53\% for parents, and 64 and 36\% for spouses. Longitudinal phenotyping by survey includes assessment of depressive symptoms (multiple instruments), anxiety, neuroticism, and other personality measures. Additionally, subjects are asked about life events, lifestyle, education, health, and religious background.

From the group of NTR participants for whom both survey data and biological samples were available, we selected controls for the MDD cases. Controls never scored high $(>0.65)$ on a general factor score for anxious depression. The factor score is a combined measure of neuroticism, anxiety, and depressive symptoms assessed via longitudinal questionnaires. ${ }^{39}$ It has a mean of 0 and a SD of 0.7. Subjects never reported a history of MDD in any survey or at the blood sampling visit (either as a complaint for which treatment was sought from a specialist, reported medication use, or via the CIDI). Controls and their parents were born in the Netherlands or western Europe. If there were multiple eligible controls in a family, we first matched on sex and age, and used the highest number of completed questionnaires as an additional criterion. Only biologically unrelated subjects were selected.

The NESDA controls (133 from general practice, 24 from Adolescents at Risk for Anxiety and Depression Study) came from a larger healthy control group. Controls did not have a lifetime diagnosis of MDD or an anxiety disorder as assessed by the CIDI, and all controls reported low depressive symptoms at baseline ( $<16$ on K-10 (29), $<$ four on Inventory of Depressive Symptoms $\left.{ }^{41}\right)$.

\section{IRB approvals and informed consent}

The NESDA and NTR studies were approved by the Central Ethics Committee on Research involving human subjects of the VU University Medical Center, Amsterdam, an Institutional Review Board certified by the US Office of Human Research Protections (IRB number IRB-2991 under Federal wide Assurance-3703; IRB/institute codes, NESDA 03-183; NTR 03-180). All subjects provided written informed consent. As part of the GAIN application process, consent forms were re-reviewed. For NESDA, only 22 respondents refused informed consent for genetic research
(1.7\% of all respondents approached). NTR participants were approached with a broad consent form for the entire NTR biobank project.

\section{Phenotyping \\ MDD, depressive symptoms, and other psychopathology indicators}

NESDA The Composite International Diagnostic Interview, section E, version $2.1^{36}$ was used to diagnose MDD. The interview also provides information on age of onset, number of episodes of MDD, and specific symptoms of depression. Information on lifetime comorbid panic disorder with or without agoraphobia, generalized anxiety disorder, social phobia, alcohol use, and dependence was also collected with the CIDI. Depression and anxiety severity indicators include the Inventory of Depressive Symptoms-self-report (IDS-SR ${ }^{41}$ ), the Fear Questionnaire ${ }^{42}$ and the Beck Anxiety Inventory. ${ }^{43}$ Neuroticism, an endophenotype for MDD, was assessed with the NEO. ${ }^{44}$ The family tree inventory was used to examine depression in first degree relatives.

NTR Phenotyping for depression in the NTR survey studies took place with the Beck depression inventory $\left(\mathrm{BDI}^{45}\right)$ in 1993 and 1997 and with the depression scale from the YASR ${ }^{46}$ in 1991, 1995, 1997, and 2000. Neuroticism was assessed with the $\mathrm{ABV}^{47}$ in five out of six surveys (not in 1995) and with the $\mathrm{NEO}^{44}$ in 2004. Anxiety $\left(\mathrm{STAI}^{48}\right.$ ) was assessed at five out of seven surveys (not in 1995 and 2004). NTR cases and a subsample of controls underwent the CIDI protocol by phone; either as part of an earlier study ${ }^{49}$ on the heritability of major depression and anxiety disorders or as part of the selection procedure for GAIN.

\section{Biobank procedures and DNA isolation}

Before the start of the NESDA and NTR biological sample collection, processing and storage protocols were harmonized. Blood sampling for the NESDA participants took place during the baseline visit and was done between 0830-0930 $\mathrm{h}$ at one of the seven field sites, all at walking distance of laboratories facilities for immediate processing.

For NTR, biological samples were taken at the respondents' home between 0700 and $1000 \mathrm{~h}$. Starting in 2004, adult participants registered with the NTR were invited by letter to participate in a project in which blood and morning urine samples would be collected. Eligible participants were $\geq 18$ years, had returned at least one survey or came from a family in which at least one person completed a questionnaire. The letter was followed by a telephone call to schedule an appointment for a home visit. With fertile women, an appointment was made for the third to fifth day of the menstrual cycle when possible. For women taking oral contraceptives, an appointment 
was made for their pill-free week. In twin individuals, we also took buccal swabs for DNA isolation ${ }^{50}$ as blood group chimerism in twins is not rare. ${ }^{51}$ Tubes were stored, as appropriate, in melting ice $\left(0-2^{\circ} \mathrm{C}\right)$ during transport, at room temperature, or in an insulated box with a constant temperature of $37^{\circ} \mathrm{C}$. Effects of transportation and storage on blood quality, RNA with and without challenge, lymphocytes and other parameters were examined in a pilot project ${ }^{52}$ and appear to be negligible.

All venous blood samples were taken after overnight fasting using a safety-lock butterfly needle. For NESDA participants, a total of 10 blood tubes were drawn in the following order: $3 \times 7 \mathrm{ml}$ and $1 \times 2 \mathrm{ml}$ EDTA, $2 \times 7 \mathrm{ml}$ and $1 \times 2 \mathrm{ml}$ Heparin, $1 \times 4.5 \mathrm{ml} \mathrm{CTAD}, 1 \times 2 \mathrm{ml} \mathrm{NaF}$, and $1 \times 6 \mathrm{ml} \mathrm{ACD}$. For the NTR participants, a total of seven blood tubes were drawn in the following order: $2 \times 9 \mathrm{ml}$ EDTA, $2 \times 9 \mathrm{ml}$ Heparin, $1 \times 4.5 \mathrm{ml} \mathrm{CTAD,} 1 \times 7 \mathrm{ml}$ serum, and $1 \times 2 \mathrm{ml}$ EDTA. For DNA isolation blood collected in the EDTA anticoagulant tubes was used. For DNA isolation

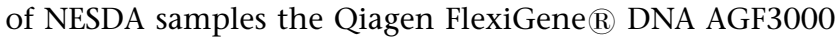
Kit for large volumes of fresh whole human blood was used on an AutoGenFlex 3000 workstation (Autogen, Holliston, MA, USA). The GENTRA Puregene $\mathbb{R}$ DNA isolation kit (manual) was used on frozen whole blood samples of the NTR. DNA concentrations were determined using the

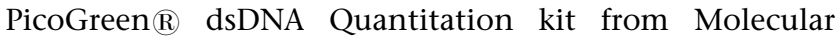
Probes. All procedures were performed according to the manufacturer's protocols.

\section{GAIN sample}

For the case and the control groups we selected unrelated subjects. Baseline characteristics of cases and controls, and of an additional NTR comparison group, are summarized in Tables 1 and 2. Tables 1 and 2 describe the data as deposited on the dBGaP website (www.ncbi.nlm.nih.gov/dbgap, 9 October 2007). After excluding subjects with genotyping or sample problems, data from 1821 MDD cases and 1822 controls are available for analyses. The actual numbers for genome-wide association analyses may turn out to be slightly smaller, as extra analyses of the genotype data may lead to removal of additional subjects. For both MDD cases and for controls there is information available on the website about demographics, personality traits (eg, neuroticism), and lifestyle (eg, smoking and alcohol use). The phenotype information for NESDA respondents is at present mainly from the baseline interview data. The baseline interview took $4 \mathrm{~h}$ on average and consisted of the CIDI, a series of self-report questionnaires, a medical interview, and a fasting blood draw. For NTR respondents, a total of up to seven surveys were mailed to the participating twin families over a period of 15 years. NTR respondents who took part in the biobank protocol were

Table 2 Characteristics of NTR comparison sample and GAIN controls

\begin{tabular}{lcc}
\hline & $\begin{array}{c}\text { NTR comparison } \\
\text { sample } \mathrm{N}=4111\end{array}$ & $\begin{array}{c}\text { GAIN controls } \\
\mathrm{N}=1822^{\mathrm{a}}\end{array}$ \\
\hline Age (years) & $43.6 \pm 15.3$ & $\begin{array}{c}45.1 \\
\text { Female, \% }\end{array}$ \\
Educational level (\% low/ & $9.2 / 61.7 / 29.2$ & $5.7 / 56.2 / 38.1$ \\
middle/high) & & 61.8 \\
With partner, \% & 82.9 & 87.0 \\
Smoking (current), \% & 37.9 & 20.4 \\
Alcohol use (last year), \% & 79.4 & 80.0 \\
NEO neuroticism & $31.7 \pm 6.3$ & $28.2 \pm 5.5$ \\
ABV neuroticism & $49.9 \pm 25.3$ & $36.7 \pm 16.0$ \\
ABV somatic complaints & $17.9 \pm 5.6$ & $15.9 \pm 3.8$ \\
STAl trait anxiety & $34.1 \pm 8.8$ & $30.1 \pm 5.6$ \\
YASR anxious depression & $5.0 \pm 4.2$ & $3.22 \pm 2.7$ \\
Beck depression & $2.1 \pm 2.8$ & $1.11 \pm 1.4$ \\
\hline
\end{tabular}

For continuous traits, the mean score and SD are listed.

${ }^{\mathrm{a}} 158$ controls are from NESDA.

Table 1 Characteristics of MDD cases by setting and total MDD group

\begin{tabular}{|c|c|c|c|c|}
\hline & \multicolumn{3}{|c|}{ MDD cases by setting } & \multirow{2}{*}{$\begin{array}{c}\text { Total MDD cases } \\
\mathrm{N}=1821\end{array}$} \\
\hline & Community $\mathrm{N}=446^{\mathrm{a}}$ & Primary care $\mathrm{N}=772$ & Mental health care $\mathrm{N}=603$ & \\
\hline Age (years) & $41.2 \pm 13.9$ & $45.9 \pm 11.7$ & $37.9 \pm 11.4$ & $42.1 \pm 12.7$ \\
\hline Educational level (\% low/middle/high) & $7.6 / 62.7 / 29.6$ & $7.0 / 54.5 / 38.5$ & $8.1 / 66.2 / 25.8$ & $7.5 / 60.4 / 32.1$ \\
\hline With partner, \% & 72.5 & 69.8 & 63.8 & 68.5 \\
\hline Smoking (current), \% & 40.1 & 39.2 & 47.3 & 42.1 \\
\hline Alcohol use (last year), \% & 63.7 & 67.5 & 66.7 & 66.3 \\
\hline Depression; age of onset & $26.8 \pm 12.5$ & $29.1 \pm 12.7$ & $25.8 \pm 11.6$ & $27.5 \pm 12.3$ \\
\hline IDS depression ${ }^{\mathrm{b}}$ & $17.2 \pm 11.3$ & $23.8 \pm 12.1$ & $33.0 \pm 12.7$ & $25.8 \pm 13.5$ \\
\hline BAl anxiety ${ }^{b}$ & $8.2 \pm 7.5$ & $13.2 \pm 9.4$ & $18.9 \pm 11.5$ & $14.3 \pm 10.6$ \\
\hline
\end{tabular}

For continuous traits, the mean score and SD are listed.

${ }^{a}$ Community cases (446): 214 from NEMESIS, 95 from ARIADNE, 137 from NTR.

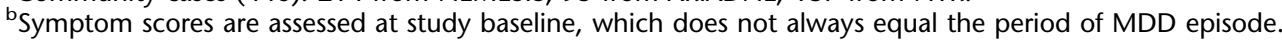


visited at home for an additional medical interview, a fasting blood draw, and collection of urine samples.

Table 1 presents the phenotype data for MDD cases, as a function of the recruitment setting, and for the total group. Patients from mental care are on average somewhat younger, have less often attained a higher educational level, less often have a spouse/partner and tend to smoke more heavily. Their profiles on the neuroticism and depression scales are more unfavourable. From comparing the total MDD group to the GAIN control group in Table 2, it may be seen that the control group is slightly older, better educated, and more often with partner/spouse. With respect to lifestyle, MDD cases smoke more often (42 vs $20 \%$ ) but are less often current drinkers (66 vs 80\%).

Table 2 shows characteristics of GAIN controls. In addition, Table 2 provides descriptive statistics, based on the survey data, for a large group of NTR participants who are not in GAIN. The subjects in the comparison group were selected as follows: the subject was not included in GAIN and was not a family member of a GAIN participant; was 18 years or older and survey data on personality traits were available. Only unrelated persons were included in the comparison group and subjects from the comparison group were matched on age and sex with the GAIN control group. Table 2 shows that the GAIN controls are 'hypercontrols': they are better educated, are more often married, and smoke less often. Their scores on personality traits related to MDD and on depression indices from the survey data show a somewhat more favourable profile than the NTR comparison sample.

\section{Discussion}

Progress in unravelling the genetic architecture of multifactorial traits such as MDD has been slow. The reasons have been noted numerous times and include inadequacies in study design, inappropriate sample-recruitment strategy, and inadequately powered samples. ${ }^{53}$ We have tried to carefully select patients and controls who are at low liability of developing MDD. Given the sample size of our study we have $80 \%$ power to detect a QTL with genotype relative risk of 1.33 if the minor allele frequency is at least 0.40 (under the assumption of a log-additive model with a population prevalence for MDD of $15 \%^{54}$ ). At $80 \%$ power, the genotype relative risk increases to 1.38 and 1.58 if the minor allele frequency is 0.25 and 0.10 , respectively. The majority of genetic studies performed to date have had insufficient power to uncover genetic variants of small effect.

The genetic basis of liability to MDD and related disorders has been established through meta-analysis of data from twin and family studies. ${ }^{13}$ The meta-analytic heritability estimate was $37 \%$. We found in the Dutch population a comparable estimate of heritability, based on twice the correlation in dizygotic twin and sibling pairs, of $36 \%$ for major depression. ${ }^{49}$ Furthermore, no qualitative or quantitative sex differences were observed in genetic architecture, which is a promising starting point for genetic linkage and association analyses, as it allows pooling of data from men and women.

The GAIN MDD study will generate GWA data on MDD cases and controls at low risk of MDD derived from two large biobank enterprises in the Netherlands. All cases were diagnosed as having DSM-IV MDD using the CIDI psychiatric interview. Cases are a highly representative sample of those affected with this disorder in the Netherlands through ascertainment in a variety of health case settings. The control sample consisted of so-called 'hypercontrols' who were selected from the lower end of the relevant risk distribution. ${ }^{32}$ This should increase the power of the study, compared to the use of unselected control samples.

One of the major strengths of the NTR and NESDA studies is that participants are followed longitudinally and that additional phenotyping and new biological sample collection are both possible. This means that genome-wide genotype data can be used in future studies of, for example, treatment outcomes or of MDD trajectories. Also, to follow up on the independently replicated genes, detailed information is available on two major theoretical correlates of MDD, that is, hypothalamic-pituitary-adrenal axis (HPA-axis) and autonomic nervous system functioning. Additional association to such correlated biological phenotypes would give construct validity to the identified risk genotypes and point to biological mechanisms. A genotype that is associated with both depression and increased sympathetic nervous system activity, for instance, would suggest that the biological action of the gene may involve the noradrenergic synapse and its signalling cascade.

NESDA and NTR subjects have provided information on major life events, and will, in follow-up studies, also be queried about the occurrence of these events. Longitudinal data, including the course of MDD, will be available from NESDA follow-up assessments at 1, 2, 4, 6, and 8 years after baseline. For NTR participants, up to seven surveys are available and the eighth survey is scheduled for 2008 . These data can be used for Stage 2 studies, in which geneenvironment interactions are assessed. Further plans for future use of the GAIN data include data pooling metaanalytic studies of MDD and other psychiatric disorders and the detailed analyses of genotypic and phenotypic data in monozygotic twins who are MDD discordant.

\section{Acknowledgements}

We would like to acknowledge support from NWO: genetic basis of anxiety and depression (904-61-090); resolving cause and effect in the association between exercise and well-being (904-61-193); twinfamily database for behavior genomics studies (480-04-004); twin research focusing on behavior (400-05-717), Center for Medical 
Systems Biology (NWO Genomics); Spinozapremie (SPI 56-464-14192); Centre for Neurogenomics and Cognitive Research (CNCR-VU); genomewide analyses of European twin and population cohorts (EU/ QLRT-2001-01254); genome scan for neuroticism (NIMH R01 MH059160); Geestkracht program of ZonMW (10-000-1002); matching funds from universities and mental health care institutes involved in NESDA (GGZ Buitenamstel-Geestgronden, Rivierduinen, University Medical Center Groningen, GGZ Lentis, GGZ Friesland, GGZ Drenthe). Major funding for this project is from the Genetic Association Information Network of the Foundation for the US National Institutes of Health, a public-private partnership between the NIH and Pfizer Inc., Affymetrix Inc. and Abbott Laboratories.

\section{References}

1 American Psychiatric Association: Diagnostic and Statistical Manual of Mental Disorders. 4th edn. Washington, DC: American Psychiatric Association, 1994.

2 Weissman MM, Bland R, Joyce PR, Newman S, Wells JE, Wittchen H-U: Sex differences in rates of depression: cross-national perspectives. J Affect Disord 1993; 29: 77-84.

3 Piccinelli M, Wilkinson G: Outcome of depression in psychiatric settings. Br J Psychiatry 1994; 164: 297-304.

4 Wells KB, Stewart A, Hays RD et al: The functioning and wellbeing of depressed patients: results from the Medical Outcomes Study. JAMA 1989; 262: 914-919.

5 Broadhead WE, Blazer DG, George LK, Tse CK: Depression, disability days, and days lost from work in a prospective epidemiologic survey. JAMA 1990; 264: 2524-2528.

6 Judd LL, Paulus MP, Wells KB, Rapaport MN: Socioeconomic burden of subsyndromal depressive symptoms and major depression in a sample of the general population. Am J Psychiatry 1996; 153: $1411-1417$.

7 Tsuang MT, Woolson RF: Excess mortality in schizophrenia and affective disorders. Arch Gen Psychiatry 1978; 35: 1181-1185.

8 Berglund M, Nilsson K: Mortality in severe depression: a prospective study including 103 suicides. Acta Psychiat Scand 1987; 76: 372-380

9 Black DW, Winokur G, Nasrallah A: Is death from natural causes still excessive in psychiatric patients? J Nerv Ment Dis 1987; 175: 674-680.

10 Zilber N, Schufman N, Lerner Y: Mortality among psychiatric patients - the groups at risk. Acta Psychiat Scand 1989; 79: $248-256$

11 Greenberg PE, Stiglin LE, Finkelstein SN, Berndt ER: The economic burden of depression in 1990. J Clin Psychiatry 1993; 54: 405-418.

12 Murray CJL, Lopez AD: Evidence-based health policy: lessons from the Global Burden of Disease Study. Science 1996; 274: $740-743$.

13 Sullivan PF, Neale MC, Kendler KS: Genetic epidemiology of major depression: review and meta-analysis. Am J Psychiatry 2000; 157: $1552-1562$.

14 Nurnberger Jr JI, Foroud T, Flury L et al: Evidence for a locus on chromosome 1 that influences vulnerability to alcoholism and affective disorder. Am J Psychiatry 2001; 158: 718-724.

15 McGuffin P, Knight J, Breen G et al: Whole genome linkage scan of recurrent depressive disorder from the depression network study. Hum Mol Genet 2005; 14: 3337-3345.

16 Holmans P, Weissman MM, Zubenko GS et al: Genetics of recurrent early-onset major depression (GenRED): final genome scan report. Am J Psychiatry 2007; 164: 248-258.

17 Zubenko GS, Maher B, Hughes III HB et al: Genome-wide linkage survey for genetic loci that influence the development of depressive disorders in families with recurrent, early-onset, major depression. Am J Med Genet B Neuropsychiatr Genet 2003; 123: $1-18$.
18 Holmans P, Zubenko GS, Crowe RR et al: Genomewide significant linkage to recurrent, early-onset major depressive disorder on chromosome 15q. Am J Hum Genet 2004; 74: 1154-1167.

19 Camp NJ, Lowry MR, Richards RL et al: Genome-wide linkage analyses of extended Utah pedigrees identifies loci that influence recurrent, early-onset major depression and anxiety disorders. Am J Med Genet B Neuropsychiatr Genet 2005; 135: 85-93.

20 Cloninger CR, Van Eerdewegh P, Goate A et al: Anxiety proneness linked to epistatic loci in genome scan of human personality traits. Am J Med Genet 1998; 81: 313-317.

21 Fullerton J, Cubin M, Tiwari $\mathrm{H}$ et al: Linkage analysis of extremely discordant and concordant sibling pairs identifies quantitativetrait loci that influence variation in the human personality trait neuroticism. Am J Hum Genet 2003; 72: 879-890.

22 Nash MW, Huezo-Diaz P, Williamson RJ et al: Genome-wide linkage analysis of a composite index of neuroticism and moodrelated scales in extreme selected sibships. Hum Mol Genet 2004; 13: $2173-2182$.

23 Neale BM, Sullivan PF, Kendler KS: A genome scan of neuroticism in nicotine dependent smokers. Am J Med Genet B Neuropsychiatr Genet 2004; 132B: $65-69$.

24 Kuo PH, Neale MC, Riley BP et al: A genome-wide linkage analysis for the personality trait neuroticism in the Irish affected sib-pair study of alcohol dependence. Am J Med Genet B Neuropsychiatr Genet 2007; 144: 463-468.

25 Willis-Owen SA, Turri MG, Munafo MR et al: The serotonin transporter length polymorphism, neuroticism, and depression: a comprehensive assessment of association. Biol Psychiatry 2005; 58: $451-456$.

26 Munafo MR, Clark T, Flint J: Does measurement instrument moderate the association between the serotonin transporter gene and anxiety-related personality traits? A meta-analysis. Mol Psychiatry 2005; 10: 415-419.

27 Middeldorp CM, de Geus EJ, Beem AL et al: Family based association analyses between the serotonin transporter gene polymorphism (5-HTTLPR) and neuroticism, anxiety and depression. Behav Genet 2007; 37: 294-301.

28 Klein RJ, Zeiss C, Chew EY et al: Complement factor $\mathrm{H}$ polymorphism in age-related macular degeneration. Science 2005; 308: 385-389.

29 Frayling TM, Timpson NJ, Weedon MN et al: A common variant in the FTO gene is associated with body mass index and predisposes to childhood and adult obesity. Science 2007; 316: 889-894.

30 Grant SF, Thorleifsson G, Reynisdottir I et al: Variant of transcription factor 7-like 2 (TCF7L2) gene confers risk of type 2 diabetes. Nat Genet 2006; 38: 320-323.

31 Duerr RH, Taylor KD, Brant SR et al: A genome-wide association study identifies IL23R as an inflammatory bowel disease gene. Science 2006; 314: 1461-1463.

32 Wellcome Trust Case Control Consortium: Genome-wide association study of 14000 cases of seven common diseases and 3000 shared controls. Nature 2007; 447: 661-678.

33 The GAIN Collaborative Research Group: New models of collaboration in genome-wide association studies: the Genetic Association Information Network. Nat Genet 2007; 39: $1045-1051$.

34 Penninx BW, Beekman AT, Smit JH et al: The Netherlands Study of Depression and Anxiety (NESDA): rationales, objectives and methods. Int J Meth Psychiatr Res, provisionally accepted. (in press).

35 Kessler RC, Barker PR, Colpe LJ et al: Screening for serious mental illness in the general population. Arch Gen Psychiatry 2003; 60: 184-189.

36 World Health Organization: Composite International Diagnostic Interview, Core Version 2.1: Interviewer's Manual. Sydney, Australia: World Health Organization, 1997.

37 Bijl RV, van Zessen G, Ravelli A, de Rijk C, Langendoen Y: The Netherlands Mental Health Survey and Incidence Study (NEMESIS): objectives and design. Soc Psychiatry Psychiatr Epidemiol 1998; 33: 581-586. 
38 Landman-Peeters KM, Hartman CA, van der Pompe G, den Boer JA, Minderaa RB, Ormel J: Gender differences in the relation between social support, problems in parent-offspring communication, and depression and anxiety. Soc Sci Med 2005; 60: $2549-2559$.

39 Boomsma DI, Beem AL, Berg M van den et al: Netherlands twin family study of anxious depression (NETSAD). Twin Res 2000; 3: $323-334$

40 Boomsma DI, de Geus EJC, Vink JM et al: Netherlands Twin Register: from twins to twin families. Twin Res Hum Genet 2006; 9: 849-857.

41 Rush AJ, Gullion CM, Basco MR, Jarrett RB, Trivedi MH: The inventory of depressive symptomatology (IDS): psychometric properties. Psychol Med 1996; 26: 477-486.

42 Marks IM, Matthews A: Brief standard self-rating for phobic patients. Behavior Res Therapy 1979; 17: 263-267.

43 Beck AT, Epstein N, Brown G, Steer RA: An inventory for measuring clinical anxiety: psychometric properties. $J$ Consult Clin Psychol 1988; 56: 893-897.

44 Costa Jr PT, McCrae RR: Domains and facets: hierarchical personality assessment using the revised NEO personality inventory. J Pers Assess 1995; 64: 21-50.

45 Beck AT, Ward CH, Mendelson M, Mock J, Erbaugh J: An inventory measuring depression. Arch Gen Psychiatry 1961; 4: $53-63$.
46 Achenbach TM: The Young Adult Self Report. Burlington, VT: University of Vermont, Dept of Psychiatry, 1990.

47 Wilde GJS: Neurotische labiliteit gemeten volgens de vragenlijstmethode (The questionnaire method as a means of measuring neurotic instability). Amsterdam: Van Rossen, 1970.

48 Spielberger CD, Gorsuch RL, Lushene RE: STAI Manual for the State-Trait Anxiety Inventory. Palo Alto, CA: Consulting Psychologists Press, 1970.

49 Middeldorp CM, Birley AJ, Cath DC et al: Familial clustering of major depression and anxiety disorders in Australian and Dutch twins and siblings. Twin Res Hum Genet 2005; 8: 609-615.

50 Min JL, Lakenberg N, Bakker-Verweij M et al: High microsatellite and SNP genotyping success rates established in a large number of genomic DNA samples extracted from mouth swabs and genotypes. Twin Res Hum Genet 2006; 9: 501-506.

51 Van Dijk BA, Boomsma DI, Man AJM de: Blood group chimerism in human multiple births is not rare. Am J Med Genet 1996; 61: $264-268$.

52 Hoekstra C, Meijer P, Kluft C et al: Genetics of dizygotic twinning. A feasibility study for a biobank. Twin Res 2004; 7: 556-563.

53 Hattersley AT, McCarthy MI: What makes a good genetic association study? Lancet 2005; 366: 1315-1323.

54 Gauderman WJ, Morrison JM: QUANTO 1.1: a computer program for power and sample size calculations for genetic-epidemiology studies, 2006, http://hydra.usc.edu/gxe. 